\title{
Interfacial Complexes between a Protein and Lipophilic Ions at an Oil-Water Interface
}

\author{
Rune A. Hartvig, ${ }^{\dagger}$ Manuel A. Méndez, ${ }^{\ddagger}$ Marco van de Weert, ${ }^{\dagger}$ Lene Jorgensen, ${ }^{\dagger}$ \\ Jesper Østergaard, ${ }^{\dagger}$ Hubert H. Girault, ${ }^{* *}$ and Henrik Jensen*,†
}

\begin{abstract}
Department of Pharmaceutics and Analytical Chemistry, Faculty of Pharmaceutical Sciences, University of Copenhagen, Universitetsparken 2, DK-2100 Copenhagen, Denmark, and Laboratoire d'Electrochimie Physique et Analytique, Ecole Polytechnique Fédérale de Lausanne (EPFL), Station 6, CH-1015 Lausanne, Switzerland
\end{abstract}

The interaction between an intact protein and two lipophilic ions at an oil-water interface has been investigated using cyclic voltammetry, impedance based techniques and a newly developed method in which the biphasic oil-water system is analyzed by biphasic electrospray ionization mass spectrometry (BESI-MS), using a dualchannel electrospray emitter. It is found that the protein forms interfacial complexes with the lipophilic ions and that it specifically requires the presence of the oil-water interface to be formed under the experimental conditions. Furthermore, impedance based techniques and BESI-MS with a common ion to polarize the interface indicated that the Galvani potential difference across the oil-water interface significantly influences the interfacial complexation degree. The ability to investigate protein-ligand complexes formed at polarized liquid-liquid interfaces is thus a new analytical method for assessing potential dependent interfacial complexation using a structure elucidating detection principle.

The behavior of biological macromolecules at liquid-liquid interfaces has important implications in the pharmaceutical sciences. It is for example well-known that proteins adsorb at a wide variety of interfaces, ${ }^{1-4}$ a process that can have unwanted consequences such as a loss of therapeutic activity due to denaturation and aggregation. ${ }^{4,5}$ Two-phase systems are at equilibrium generally associated with an interfacial potential difference, ${ }^{6}$ in the following referred to as the Galvani potential difference between a water (w) and oil (o) phase at equilibrium; $\Delta_{0}^{\mathrm{w}} \phi$. The value of $\Delta_{0}^{\mathrm{w}} \phi$ can influence adsorption kinetics and/ or adsorption isotherms. ${ }^{7}$ Electrochemistry at Interfaces between two immiscible electrolyte solutions (ITIES) provides an effective methodology for studying potential dependent

* To whom correspondence should be addressed. E-mail: hj@farma.ku.dk (H.J.); hubert.girault@epfl.ch (H.H.G.).

$\dagger$ University of Copenhagen.

* Ecole Polytechnique Fédérale de Lausanne (EPFL)

(1) Dickinson, E. J. Chem. Soc., Faraday Trans. 1998, 94, 1657-69.

(2) Walstra, P.; Deroos, A. L. Food Rev. Int. 1993, 9, 503-25.

(3) Kleijn, M.; Norde, W. Heterog. Chem. Rev. 1995, 2, 157-72.

(4) Burke, C. J.; Steadman, B. L.; Volkin, D. B.; Tsai, P. K.; Bruner, M. W.; Middaugh, C. R. Int. J. Pharm. 1992, 86, 89-93.

(5) Sluzky, V.; Tamada, J. A.; Klibanov, A. M.; Langer, R. Proc. Natl. Acad. Sci. U.S.A. 1991, 88, 9377-81.

(6) Kakiuchi, T. Liquid-Liquid Interfaces: Theory and Methods, 1st ed.; Volkov, A. G.; Deamer, D. W., Eds.; CRC Press, Inc.: Boca Raton, 1996; Chapter 1. adsorption and ion transfer processes. ${ }^{8-12}$ In relation to protein research, electrochemistry at ITIES have been used to study protein adsorption, ${ }^{13}$ protein adsorption kinetics, ${ }^{7}$ the effects on transfer of aqueous ions, ${ }^{14}$ and the assisted transfer of proteins to the oil phase. ${ }^{15-18}$ Recently, electrochemistry at ITIES has been used for detecting proteins in solution by means of a protein assisted transfer of organic anions into the water phase at positive potentials. ${ }^{19-22}$ A suggested mechanism for the organic anion transfer involves the formation of a protein-anion complex at the interface. $^{22}$

Usually electrospray ionization mass spectrometry (ESI-MS) involves an aqueous solution which by application of a high voltage is sprayed into a mass analyzer. Recently, the development of a new biphasic electrospray interface has allowed interfacial complexes in two phase systems to be analyzed using a mass spectrometer; the new technique is termed biphasic electrospray mass spectrometry (BESI-MS). ${ }^{23-26}$ The combined use of BESIMS and electrochemistry at ITIES is attractive as it allows studying quantitative mechanistic aspects of adsorption and ion transfer processes as well as structural characteristics of complexes formed at the interface. However, the usual experimental conditions in

(7) Thomsen, A. E.; Jensen, H.; Jorgensen, L.; van de Weert, M.; Østergaard, J. Colloid. Surface. B 2008, 63, 243-48.

(8) Arrigan, D. W. M. Anal. Lett. 2008, 41, 3233-52.

(9) Vanysek, P.; Ramirez, L. B. J. Chil. Chem. Soc. 2008, 53, 1455-63.

(10) Reymond, F.; Fermin, D.; Lee, H. J.; Girault, H. H. Electrochim. Acta 2000, 45, 2647-62.

(11) Samec, Z. Pure Appl. Chem. 2004, 76, 2147-80.

(12) Vanysek, P. Electrochim. Acta 1995, 40, 2841-47.

(13) Vanysek, P.; Sun, Z. J. Electroanal. Chem. 1990, 298, 177-94.

(14) Vanysek, P.; Reid, J. D.; Buck, R. P. J. Electrochem. Soc. 1984, 131, C107.

(15) Vagin, M. Y.; Trashin, S. A.; Ozkan, S. Z.; Karpachova, G. P.; Karyakin, A. A. J. Electroanal. Chem. 2005, 584, 110-16.

(16) Vagin, M. Y.; Malyh, E. V.; Larionova, N. I.; Karyakin, A. A. Electrochem. Commun. 2003, 5, 329-33.

(17) Osakai, T.; Shinohara, A. Anal. Sci. 2008, 24, 901-06.

(18) Shinshi, M.; Sugihara, T.; Osakai, T.; Goto, M. Langmuir 2006, 22, 593744.

(19) Herzog, G.; Moujahid, W.; Strutwolf, J.; Arrigan, D. W. M. Analyst 2009, 134, 1608-13.

(20) Herzog, G.; Kam, V.; Arrigan, D. W. M. Electrochim. Acta 2008, 53, 720409.

(21) Kivlehan, F.; Lanyon, Y. H.; Arrigan, D. W. M. Langmuir 2008, 24, 987682.

(22) Scanlon, M. D.; Jennings, E.; Arrigan, D. W. M. Phys. Chem. Chem. Phys. 2009, 11, 2272-80.

(23) Prudent, M.; Mendez, M. A.; Girault, H. H. Anal. Sci. 2008, 24, 1399-404.

(24) Mendez, M. A.; Prudent, M.; Su, B.; Girault, H. H. Anal. Chem. 2008, 80, 9499-507.

(25) Oyama, H.; Ohashi, A.; Watarai, H. Anal. Sci. 2004, 20, 1543-47.

(26) Watarai, H.; Matsumoto, A.; Fukumoto, T. Anal. Sci. 2002, 18, 367-68. 


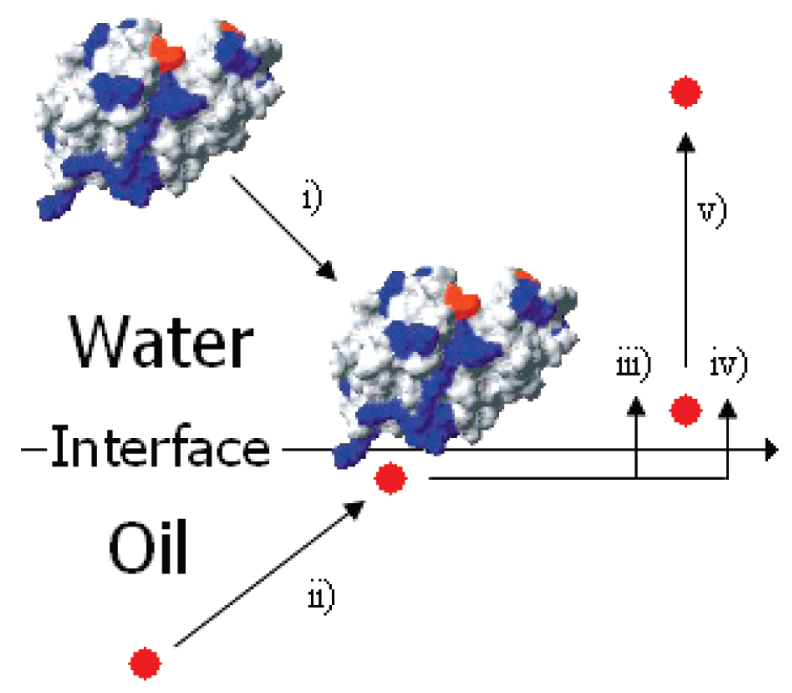

Figure 1. The red circle represents the organic anion $\left(\mathrm{TPBCl}^{-}\right.$or TPFB $^{-}$), the colored groups on lysozyme represents positively (blue) and negatively (red) charged amino acids at the investigated $\mathrm{pH}$ value of 2.81, as calculated from reference. ${ }^{27}$ The horizontal line corresponds to the interface separating water (top) and 1,2-DCE (bottom) and also represent increasing interfacial potential difference in the direction to the right. In the order of increasing $\Delta_{\circ}^{\mathrm{w}} \phi$ the events depicted thus correspond to, (i) adsorption of lysozyme which happens within the entire potential window, but increasingly so at $\Delta_{0}^{\mathrm{w}} \phi>\mathrm{pzc}$, as evident from capacitance scans (ii) formation of an interfacial complex between lysozyme and one or more organic anion molecules, as investigated by BESI-MS (iii) assisted transfer of the organic anion to the water phase where it is bound to adsorped lysozyme, gives rise to the forward prepeak in the cyclic voltammogram (iv) the forward main peak is the result of an assisted ion transfer which is less favorable, and which ultimately leads to precipitation of lysozyme (v) the complex then may diffuse to the bulk phase.

electrochemistry (high salt concentration) and BESI-MS (volatile buffer systems) are not compatible, and great care should thus be taken when combining data using the two techniques.

In this work we have studied the electrochemical responses obtained at an ITIES under conditions which are directly compatible with those used in a BESI-MS experiment. Further, we demonstrate the utility of BESI-MS with proteins and show for the first time the direct detection of a complex formed between an intact protein and small ionic species at a liquid-liquid interface. The effects of different Galvani potential differences on the adsorption of lysozyme are also investigated and the correlation between potential difference controlled protein adsorption and complex formation is investigated by means of an imposed distribution potential in the BESI-MS. The proposed model is summarized in Figure 1.

\section{EXPERIMENTAL SECTION}

Reagents. Deionized water $(18.2 \mathrm{M} \Omega \mathrm{cm})$ was obtained from a Millipore Milli-Q system (Millipore, Bedford, MA) and acetic acid from Sigma (St Louis, MO). Lysozyme and the organic solvent 1,2-dichloroethane (1,2-DCE) were obtained from Fluka Chemie (Buchs, Switzerland). The organic salts BTPPATPBCl and BTPPATPFB were synthesized from bis(triphenylphosphoranylidene)ammonium chloride (BTPPACl) (Fluka Chemie) and either potassium tetrakis(4-chlorophenyl) borate (KTPBCl) (Fluka

(27) Kuramitsu, S.; Hamaguchi, K. J. Biochem. 1980, 87, 1215-19.
Chemie) or lithium tetrakis(pentafluorophenyl)borate (LiTPFB) (Boulder Scientific Company, Mead, CO), respectively.

Electrochemical Setup. Voltammetric and potential scan methods were carried out as previously described, ${ }^{7}$ using a four electrode potentiostat PGSTAT30 (Autolab, Eco Chemie B.V., Utrecht, The Netherlands) and a custom-made glass cell from EuroGlas (Karlslunde, Denmark). The surface area of the organicaqueous interface was $1.4 \mathrm{~cm}^{2}$ and the temperature was maintained at $25 \pm 0.5^{\circ} \mathrm{C}$ by placing the cell in a water jacket. The Galvani potential difference $\left(\Delta_{\circ}^{\mathrm{w}} \phi\right)$ was controlled using two $\mathrm{Ag}$ / $\mathrm{AgCl}$ reference electrodes, one in the aqueous phase and one in a reference phase in contact with the 1,2-DCE phase (Scheme 1). The two counter electrodes were made of pure platinum and situated at each side of the interface. All potentials are taken with point of reference in the aqueous phase, so that a positive potential corresponds to a more positive potential for the aqueous phase relative to the potential of the oil phase. The potential is adjusted to the Galvani potential scale by fixing the potential of zero charge (pzc) of the cell without lysozyme at $\Delta_{0}^{\mathrm{w}} \phi=0 .{ }^{28}$

The transfer of ions was measured as an increase or decrease in direct current, where a positive current corresponds to transfer of a positive charge from the aqueous phase to the organic phase, or a negative charge transferred in the opposite direction. Capacitance-potential curves were obtained by measuring the real and imaginary current response from a sinusoidal voltage amplitude of $5 \mathrm{mV}$ (rms) and a frequency of $6 \mathrm{~Hz}$ at a range of potential values. In the absence of ion transfer across the interface, the total impedance can be considered as a resistor and capacitor in series. The interfacial capacitance (C) is then obtained as follows:

$$
C=\frac{1}{\omega \times Z_{\mathrm{Im}}}
$$

where $Z_{\mathrm{Im}}$ is the imaginary part of the impedance and $\omega$ is the angular frequency calculated as the applied frequency times $2 \pi$.

Biphasic Electrospray MS. The setup used was as described previously. ${ }^{23}$ In short, the two liquids, 1,2-DCE and water, are forced through separate channels, using two $100 \mu \mathrm{L}$ syringes (Hamilton, Bonaduz, Switzerland) and a syringe pump (KdScientific, Holliston, MA). The two channels, and thus the two liquids, are joined at the tip of a polyimide microchip from DiagnoSwiss SA (Monthey, Switzerland) and sprayed onto the MS-interface at a combined flow rate of $2 \mu \mathrm{L} / \mathrm{min}$ controlled by the pump. The mass spectrometer was a LCT time-of-flight mass spectrometer (Micromass, Manchester, UK) with the commercial electrospray source being replaced by the microchip described above. The high voltage $(3.5 \mathrm{kV})$ was connected to the stainless steel needle of the syringe containing the water phase. The temperature was set at $25{ }^{\circ} \mathrm{C}$ and mass spectra were averaged over 5 min to obtain good quality spectra.

\section{RESULTS AND DISCUSSION}

Cyclic Voltammetry. Usually the experimental conditions in electrochemistry and mass spectroscopy are not compatible. Electrochemical experiments often require relatively high con-

(28) Girault, H. H. J.; Schiffrin, D. J. Electrochim. Acta 1986, 31, 1341-1342. 


\begin{tabular}{c|c|c|}
$\mathrm{Ag} /$ & Ref. phase & Oil Phase \\
$1 \mathrm{mM}$ & $5 \mathrm{mM}$ BTPPATPFB \\
$\mathrm{AgCl}$ & $\begin{array}{c}\text { BTPPACl } \\
\text { (Aq.) }\end{array}$ & or \\
& & $\begin{array}{c}\text { BTPPATPBCl } \\
(1,2-\mathrm{DCE})\end{array}$
\end{tabular} \mid

centrations of organic and inorganic ions to render the phases electrically conductive. However, ESI-MS has a low tolerance to such species as these ions will greatly reduce, or completely suppress, the signal of ions from the compound of interest. ${ }^{29}$ Therefore, the electrochemical setup was modified to closely resemble more optimal MS conditions. The composition of the aqueous phase in the MS and electrochemical experiments were kept the same: $0.5 \%$ (v/v) acetic acid $\pm 100 \mu \mathrm{M}$ lysozyme. The aqueous acetic acid solution has a measured $\mathrm{pH}$ of 2.81 corresponding to an ionic strength of $\sim 1.5 \mathrm{mM}$ which is sufficient for a polarization of the interface to be established. The electrochemical response of the cell (Scheme 1.) with and without lysozyme in the aqueous phase is shown in Figure 2.

Without lysozyme the potential window is limited by the transfer of acetate ions from the water phase to the 1,2-DCE phase at negative potentials (to the left), and by the transfer of the $\mathrm{H}^{+}$ ion from water to the 1,2-DCE phase at positive potentials. Upon addition of lysozyme to the aqueous phase the voltammogram changes markedly (Figure 2). On the forward (f) scan a prepeak (pp) is observed at $\Delta_{0}^{\mathrm{w}} \phi_{\mathrm{pp}, \mathrm{f}}=0.27 \mathrm{~V}$ followed by a main peak (p) at $\Delta_{o}^{\mathrm{w}} \phi_{p, f}=0.46 \mathrm{~V}$. On the reverse (r) scan a large negative peak is observed with a maximum at $\Delta_{0}^{\mathrm{w}} \phi_{\mathrm{p}, \mathrm{r}}=0.11 \mathrm{~V}$. The forward peak, prepeak and reverse peak has previously been observed at comparable $\mathrm{pH}$ values employing a similar proce-

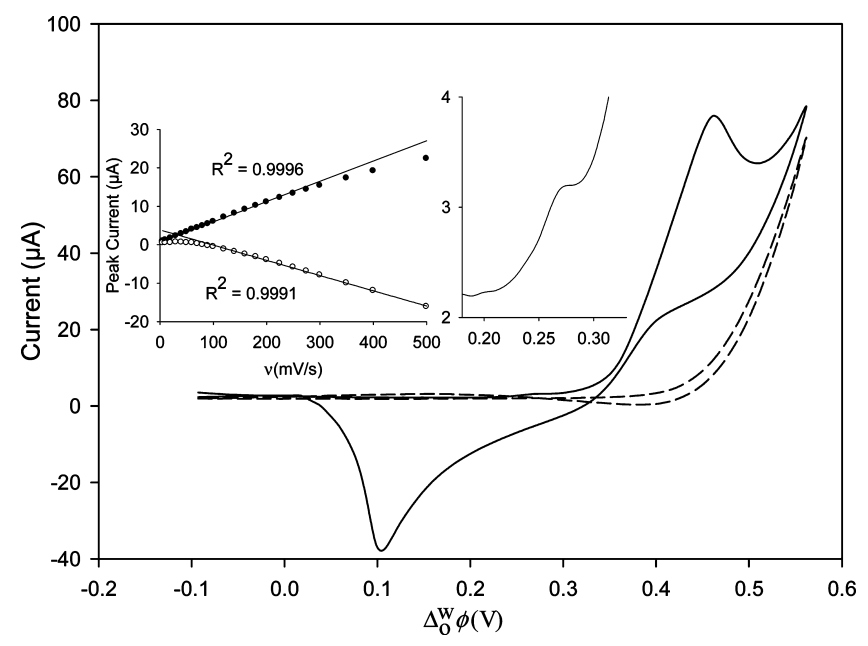

Figure 2. Cyclic voltammogram. Conditions are as described in Scheme 1, with BTPPATPBCI as the organic salt, and either with $100 \mu \mathrm{M}$ lysozyme (solid line) or without (dashed line). For the full cyclic voltammogram a comparable current response was observed using either BTPPATPBCI or BTPPATPFB as supporting electrolyte in the organic phase, whereas scan rate studies were only performed for BTPPATPBCI. insert right) is a close-up of the cyclic voltammogram with lysozyme present showing the prepeak. insert left) relation between scan rate $(\mathrm{mV} / \mathrm{s})$ and peak current $(\mu \mathrm{A})$ of the prepeak on the forward scan $(\bullet)$ and the prepeak on the reverse scan $(O)$. A linear relation is shown with scan rate for the forward prepeak in the range $5-200 \mathrm{mV} / \mathrm{s}$, and for the reverse prepeak $120-500 \mathrm{mV} / \mathrm{s}$.

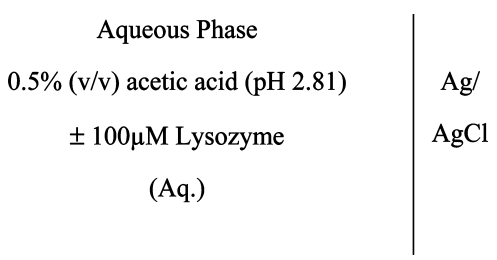

dure, but with lower concentrations of lysozyme and with $\mathrm{LiCl}$ and $\mathrm{HCl}$ as supporting electrolytes. ${ }^{22}$ In that study it was concluded that the forward main peak originated from the formation of an interfacial complex and that the dissociation of the complex could be seen as the peak in the reverse scan. ${ }^{22}$ It was also found that the peak currents of the forward and reverse peaks increased linearly with the square root of the scan rate, indicating that these processes are diffusion controlled. The current response of the prepeak increased linearly with the scan rate, which is indicative of an adsorption process. ${ }^{22}$ It was not possible to perform similar scan rate studies of the main forward and reverse peak in the present system as the observed peak currents were found to be strongly dependent on the number of repeated scans. The irreversibility between successive scans is probably due to precipitation of lysozyme, which could be seen as a white cloud at the aqueous side of the interface. Under the present conditions the precipitate was only observed after the potential had been raised beyond the onset of the forward peak (ca. $0.3 \mathrm{~V}$ in Figure 2). At least 50 scans could be repeated within the potential window without producing visible precipitate when the potential was kept lower than this value. If no external potential was forced on the interface (open circuit potential) precipitation was not observed, even $24 \mathrm{hrs}$ after lysozyme addition. By simply raising the potential from its open circuit value to $\Delta_{0}^{\mathrm{w}} \phi_{\mathrm{p}, \mathrm{f}}=0.46 \mathrm{~V}$, the formation of precipitate could be observed to occur rapidly. The events leading to precipitation therefore seem to be related to the transfer of the organic anion on the forward scan at potentials higher than that of the prepeak, as will be discussed shortly. The main peak is accountable for the majority of the transfer of anions and the observed precipitation could be the consequence of the neutralization of the charged lysozyme at high potentials by multiple $\mathrm{TPBCl}^{-}$anions. Prepeaks in cyclic voltammetry are usually associated with adsorption of the product of the charge transfer process, ${ }^{30}$ which is also the case at ITIES. Here, the prepeak suggests the transfer of $\mathrm{TPBCl}^{-}$ followed by a specific interaction with lysozyme. In order to elucidate the mechanism experimentally, the scan rate dependence on peak current may be used. These experiments presented a challenge due to precipitation at the interface at potentials greater than ca. $0.3 \mathrm{~V}$. However, if the potential is kept below ca. $\Delta_{0}^{\mathrm{w}} \phi=0.3 \mathrm{~V}$, scan rate studies can still be performed on the prepeak avoiding the irreversible influence of precipitation. While cycling the potential between the open circuit potential and $0.3 \mathrm{~V}$ two peaks are observed; (i) the prepeak on the forward scan $\left(\Delta_{o}^{\mathrm{w}} \phi_{\mathrm{pp}, \mathrm{f}}=0.27 \mathrm{~V}\right)$, as seen in the

(29) deHoffmann, E.; Stroobant, V. Mass Spectrometry: Principles and Applications, 3rd ed.; John Wiley \& Sons, Inc.: New York, 2007; Chapter 8.

(30) Bard, A. J.; Faulkner, L. R. Electrochemical Methods: Fundamentals and Applications, 2nd ed.; John Wiley \& Sons, Inc.: New York, 2001. 


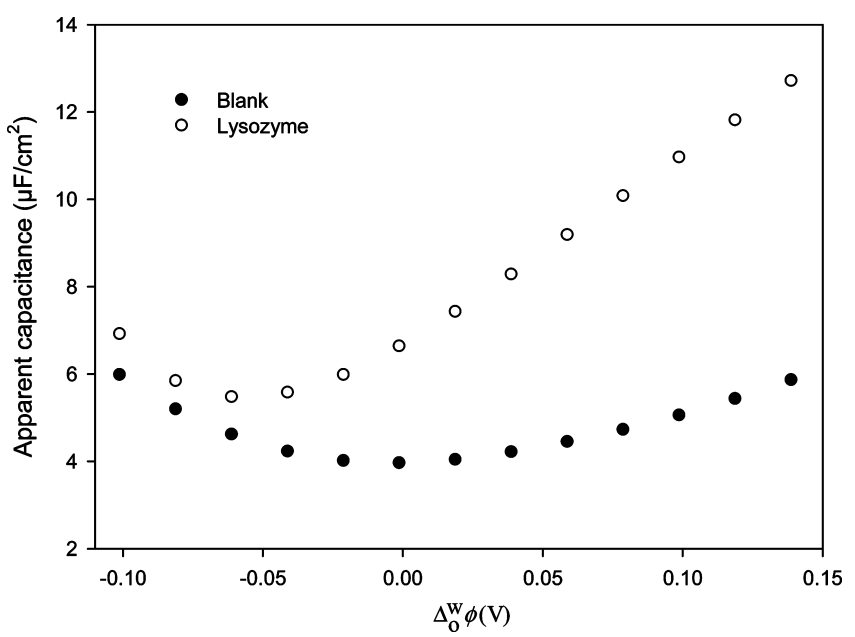

Figure 3. Apparent capacitance potential curves. Conditions as in Scheme 1, with BTPPATPBCl as organic salt and before $(\bullet)$ and after $(O)$ the addition of $100 \mu \mathrm{M}$ lysozyme.

$\mathrm{CV}$ in Figure 2, and (ii) a peak on the return scan at potential $\Delta_{0}^{\mathrm{w}} \phi_{\mathrm{pp}, \mathrm{r}}=0.17 \mathrm{~V}$, which is not observed clearly in the full voltammogram (Figure 2). The forward prepeak showed a linear peak current vs scan rate dependence in the region from 5 to 200 $(\mathrm{mV} / \mathrm{s})$ as seen from the fit in Figure 2 (left insert). This behavior is indicative of a process in which the transferred anion $\left(\mathrm{TPBCl}^{-}\right)$ is strongly adsorbed. ${ }^{31}$ As lysozyme is strongly adsorbed to this interface and is shown to form complexes with the anion in question, the adsorbed state might very well be an interaction between $\mathrm{TPBCl}^{-}$and adsorbed lysozyme, a process that in effect would give the same response. The current response of the prepeak on the reverse scan $\left(\Delta_{o}^{\mathrm{w}} \phi_{\mathrm{pp}, \mathrm{r}}=0.17\right)$ exhibit a linear relationship with scan rates higher than $100 \mathrm{mV} / \mathrm{s}$ (Figure 2, insert left) corresponding to the transfer of interfacially bound $\mathrm{TPBCl}^{-}$to the bulk 1,2-DCE phase. However the linear fit is displaced and does not intersect the origin as expected for a completely reversible process. A possible mechanism explaining this behavior could involve the desorption and diffusion to the bulk water phase of the lysozyme:anion complex. This depletion of $\mathrm{TPBCl}^{-}$from the water side of the interface results in near zero currents on the reverse scan. However, as the scan rate is raised and the time frame for each scan is lowered, the linear dependence of the peak current on the scan rate is in accordance with the transfer of adsorbed/complexed $\mathrm{TPBCl}^{-}$ to the oil phase.

Capacitance Potential Curves. Capacitance potential curves were obtained with and without lysozyme (Figure 3). Upon addition of $100 \mu \mathrm{M}$ lysozyme to the aqueous phase, the minimum of the curve, the potential of zero charge (pzc), shifts ca. $50 \mathrm{mV}$ to lower potentials. A change in pzc is an indication of specific adsorption in accordance with an Esin-Markov effect. A shift of pzc to more negative potentials is expected for accumulation of positively charged particles at the aqueous side of the interface as has been demonstrated with charged $\mathrm{TiO}_{2}$ particles. ${ }^{32}$ Lysozyme has a $\mathrm{pI}$ value of $11.16^{33}$ and thus carries a net positive charge

(31) Wopschal, R. H.; Shain, I. Anal. Chem. 1967, 39, 1514.

(32) Jensen, H.; Fermin, D. J.; Moser, J. E.; Girault, H. H. J. Phys. Chem. B 2002, 106, 10908-14.

(33) Kuehner, D. E.; Engmann, J.; Fergg, F.; Wernick, M.; Blanch, H. W.; Prausnitz, J. M. J. Phys. Chem. B 1999, 103, 1368-74. at the experimental $\mathrm{pH}$ of 2.81 , the capacitance potential results are therefore consistent with expectations. However, overall negatively charged proteins have also shown a positive shift in $\mathrm{pzc},{ }^{7,34}$ probably due to preferential adsorption of positively charged domains of the protein. From the capacitance potential scan curves it can be observed that the difference in capacitance, before and after addition of lysozyme, is greatest at potentials more positive than the pzc. In contrast, at potentials in the negative direction capacitance values change much less. The difference in capacitance values reflect a change in charge density at the surface, which suggests that the positively charged lysozyme adsorbs much more at positive potentials than at negative potentials, as would be expected for an electrostatically governed adsorption. By comparison with previous theoretical analysis ${ }^{35}$ it may be concluded that the adsorption of lysozyme is influenced by the imposed potential difference. Therefore lysozyme adsorption is favored at high potential, which is also in accordance with the scan rate studies.

Cyclic voltammetry and capacitance potential scans indicate that lysozyme adsorbs in most of the potential window and that at sufficiently high and positive potentials it will participate in assisted ion transfer of the 1,2-DCE supporting electrolyte, which in turn is involved in the precipitation of lysozyme. From these data it is not possible to finally conclude if the ion transfer involves the formation of an interfacial protein:ion complex, and details concerning the complex stochiometry of possible interfacial protein:ion complexes are unknown. We therefore utilize the BESIMS technique to investigate the existence of such complexes under the present experimental conditions.

BESI-MS of Lysozyme. Figure 4 (left) shows the BESI-MS mass spectrum of lysozyme in $0.5 \%(\mathrm{v} / \mathrm{v})$ acetic acid in contact with 1,2-DCE. The spectrum resembles those obtained for lysozyme solutions without an oil phase, obtained with the same spectrometer (data not shown) and in other laboratories. ${ }^{36}$

The spectrum is dominated by two peaks at $m / z=1432$ and $m / z=1591$ corresponding to the +10 and +9 charge states of lysozyme, respectively. From hydrogen-ion titrations an overall positive charge of around 10 for lysozyme is expected in solution at the measured $\mathrm{pH}$ of 2.81 and low ionic strength. ${ }^{33}$ However, the apparent correspondence between the expected charge in solution and measured charge on the MS is coincidental. Solution $\mathrm{pH}$ does not fix the charge state of the protein in the gas phase of the MS as can be realized from the fact that similar patterns in MS-spectra have been observed for lysozyme at both neutral and acidic $\mathrm{pH}^{36}$ and negative charge states can be observed at acidic $\mathrm{pH}$, when operating the MS in negative ion-mode. ${ }^{37}$ However, an important factor governing the charge state distribution in the MS-spectrum is the conformation of the protein in solution..$^{38,39}$ The observation of only a few peaks, serves as evidence for a folded (native) protein, while the observation of broadly distributed

(34) Georganopoulou, D. G.; Williams, D. E.; Pereira, C. M.; Silva, F.; Su, T. J.; Lu, J. R. Langmuir 2003, 19, 4977-84.

(35) Su, B.; Eugster, N.; Girault, H. H. J. Electroanal. Chem. 2005, 577, 18796.

(36) Samalikova, M.; Matecko, I.; Muller, N.; Grandori, R. Anal. Bioanal. Chem. 2004, 378, 1112-23.

(37) Konermann, L.; Douglas, D. J. J. Am. Soc. Mass Spectrom. 1998, 9, 124854.

(38) Chowdhury, S. K.; Katta, V.; Chait, B. T. J. Am. Chem. Soc. 1990, 112, 9012-13.

(39) Katta, V.; Chait, B. T. J. Am. Chem. Soc. 1991, 113, 8534-35. 


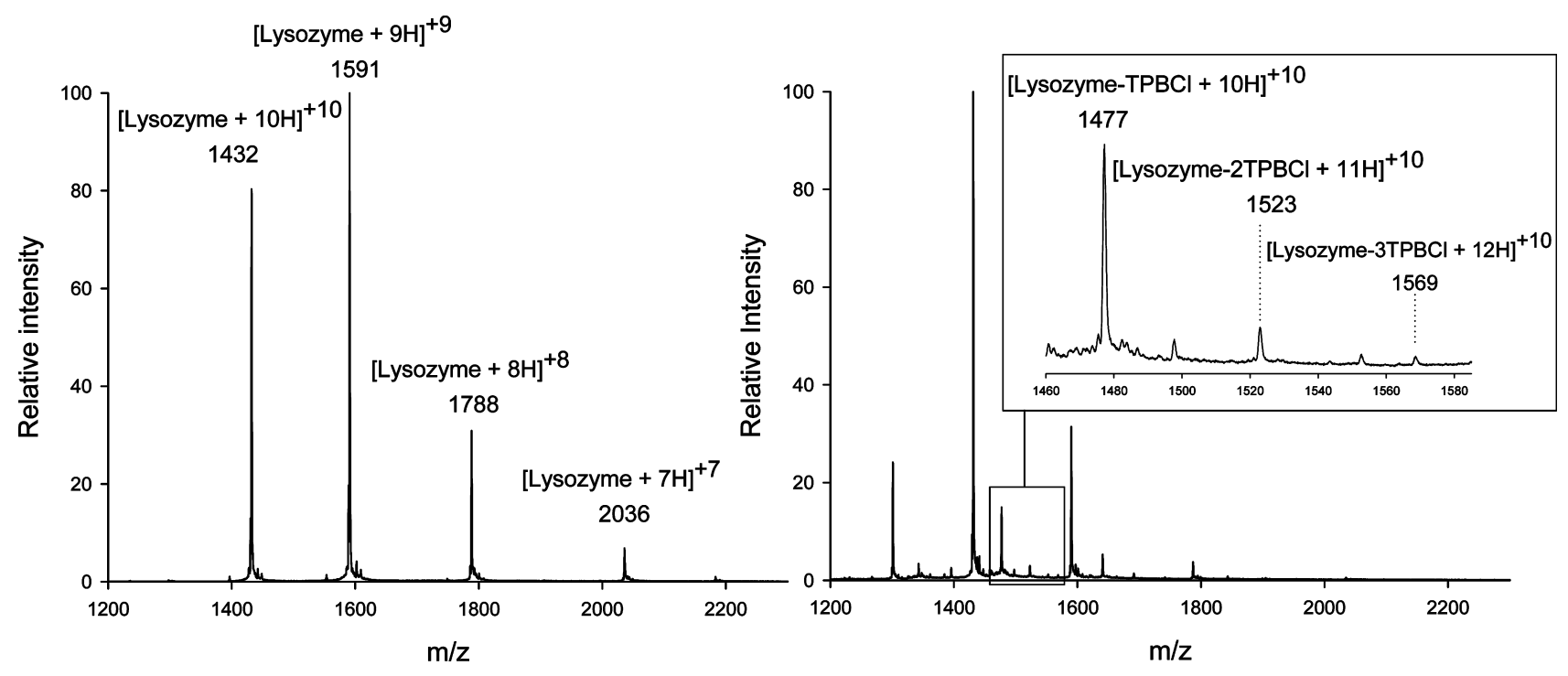

Figure 4. BESI-MS. (left) Aqueous phase: $100 \mu \mathrm{M}$ lysozyme in $0.5 \%(\mathrm{v} / \mathrm{v})$ acetic acid. Oil phase: DCE. The distribution of $m / z$ values observed is the fingerprint of a folded protein. (Right) aqueous phase: $100 \mu \mathrm{M}$ Lysozyme in $0.5 \%(\mathrm{v} / \mathrm{v})$ acetic acid. Oil phase: $10 \mu \mathrm{M}$ BTPPATPBCI in DCE. By continually adding +45.8 (corresponding to $\mathrm{TPBCl}^{-}+\mathrm{H}^{+}$) to the value of the lysozyme peak at $\mathrm{m} / \mathrm{z}=1432$, the expected value of the complexes formed between this charged lysozyme and the organic anion $\mathrm{TPBCl}^{-}$were found. 1:1, 1:2 and 1:3 complexes are observed for all major lysozyme charge states in the spectra.

charge states of high charge is an indication of denaturation. ${ }^{29}$ From these observations we conclude that lysozyme in this study was not significantly unfolded in any of the experiments.

Complexes of Lysozyme and $\mathrm{TPBCl}^{-}$. In an attempt to detect directly the complex between the protein and the organic anion $\mathrm{TPBCl}^{-}$, the anion was dissolved in 1,2-DCE as the BTPPATPBCl salt. Figure 4 (right) shows the effect of adding $10 \mu \mathrm{M}$ BTPPATPBCl to the oil phase. To the far left, outside the depicted axis, the organic cation is found $(m / z=539)$ as the peak with highest intensity. The other new peaks in the spectra are those corresponding to complexes between lysozyme and the organic anion $\mathrm{TPBCl}^{-}$. The complexes observed are in order of decreasing intensity; 1:1, 1:2, and 1:3 (lysozyme:TPBCl). To investigate if higher order complexes could be formed, the concentration of BTPPATPBCl in the organic phase was changed to $1 \mathrm{mM}$, but this did not result in additional peaks (data not shown). Care should be taken when interpreting the observed complexes on the MS as the analytes are transferred from a solution, in this case a biphasic solution, into the gas phase. The molecular interactions in solution are dominated by hydrogen-bonding, van der Waals forces and hydrophobic interactions, whereas electrostatic interactions are dominant in the gas phase. ${ }^{29,40}$ Therefore, a control experiment was performed in which an aqueous phase of $0.5 \%(\mathrm{v} / \mathrm{v})$ acetic acid, containing $10 \mu \mathrm{M}$ of the anion $\mathrm{TPBCl}^{-}$as a water-soluble salt (KTPBCl) and $100 \mu \mathrm{M}$ lysozyme was sprayed into the MS. The resulting mass spectra (data not shown) was equal to those for pure lysozyme in Figure 4 (left) and so did not contain any of the peaks assigned to lysozyme:TPBCl complexes. The fact that complexes did not form when both components were solubilized in the aqueous phase suggests that they are neither a gas phase event nor an aqueous phase event but specifically require the presence of the oil-water interface to form.

(40) Hofstadler, S. A.; Sannes-Lowery, K. A. Nat. Rev. Drug Disc. 2006, 5, 58595.
From these results it is not possible to gain information about the location of the $\mathrm{TPBCl}^{-}$binding site on lysozyme, but a tentative proposition can be made based on knowledge of the orientation of adsorbed lysozyme on other surfaces. Experiments indicate that adsorbed lysozyme orients itself in a way that results in a close proximity between the interface and several positively charged amino acids. ${ }^{41,42}$ Monte Carlo simulations for lysozyme adsorption at a mica surface support these findings and suggest that Lys1, Arg14, and Arg128 constitute the strongest interactions to the interface, based on proximity. ${ }^{43}$ It therefore seems sensible to speculate that these charged groups contribute to the binding of the organic anions by electrostatic interaction in accordance with the observation that a maximum of 3 anions are observed to be bound per lysozyme molecule.

Complexes of Lysozyme and TPFB $^{-}$. The experiments discussed above with $\mathrm{TPBCl}^{-}$were repeated using $\mathrm{TPFB}^{-}$as the organic anion and the results are shown in Figure 5. Again, new peaks are observed corresponding to formation of complexes. However complexes ranging from 1:1 to 1:5 (Lysozyme:TPFB) are found in these spectra. The results from the control experiment, where $10 \mu \mathrm{M}$ LiTPFB was solubilized in the aqueous phase with lysozyme and acetic acid, also differed in that a 1:1 complex was observed in this case (data not shown). This peak is only about $25 \%$ of the intensity of the peak observed as a 1:1 complex in the original experiment (Figure 5), when both peaks are taken as relative to their base peak at 1432 . Therefore, building on the discussion for $\mathrm{TPBCl}^{-}$, we can again conclude that the organic ion, in this case $\mathrm{TPFB}^{-}$, forms complexes with lysozyme that depend on the presence of the oil-water interface. The maximal number of $\mathrm{TPFB}^{-}$bound (1:5) is not consistent with

(41) Aizawa, T.; Koganesawa, N.; Kamakura, A.; Masaki, K.; Matsuura, A.; Nagadome, H.; Terada, Y.; Kawano, K.; Nitta, K. FEBS Lett. 1998, 422, $175-78$.

(42) Dismer, F.; Petzold, M.; Hubbuch, J. J. Chromatogr., A 2008, 1194, 1121.

(43) Mulheran, P.; Kubiak, K. Mol. Simul. 2009, 35, 561-66. 


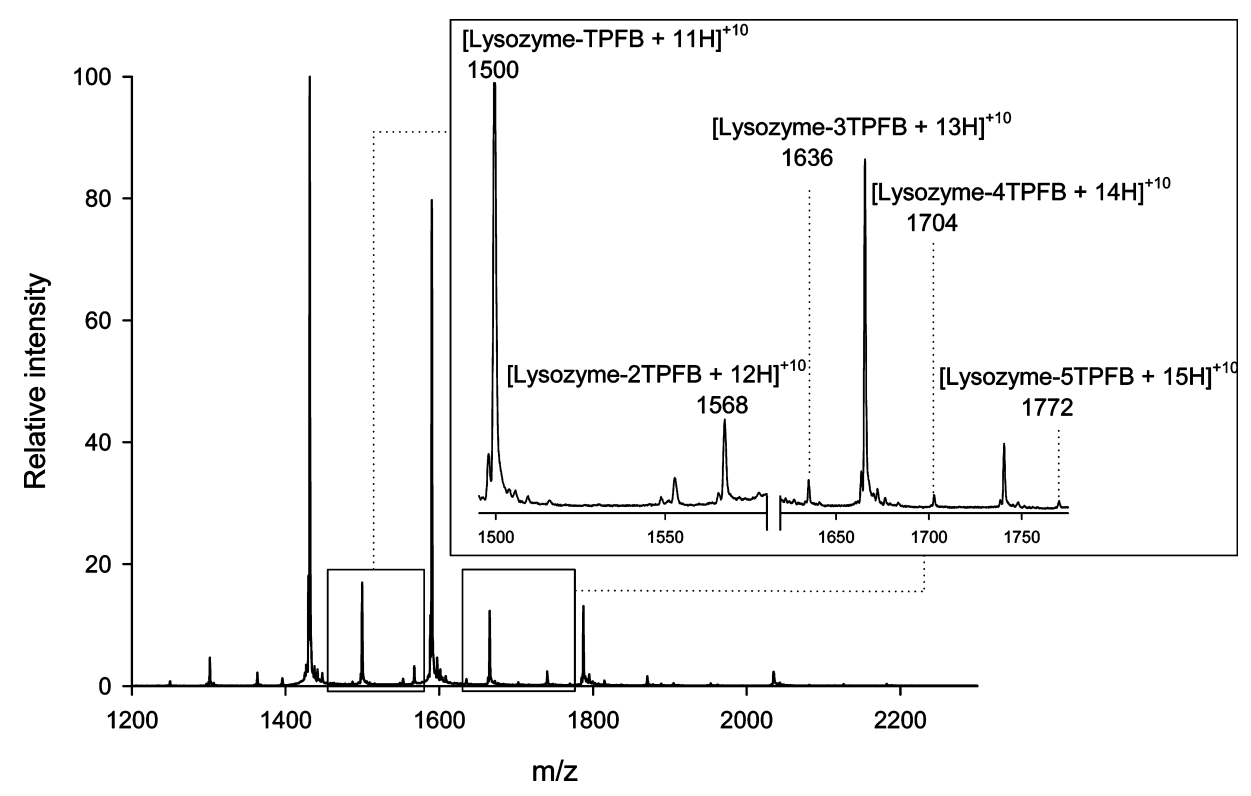

Figure 5. BESI-MS. Aqueous phase: $100 \mu \mathrm{M}$ lysozyme in $0.5 \%(\mathrm{v} / \mathrm{v})$ acetic acid. Oil phase: $10 \mu \mathrm{M}$ BTPPATPFB in DCE. By continually adding +68.0 (corresponding to TPFB ${ }^{-}+\mathrm{H}^{+}$) to the value of the lysozyme peak at $\mathrm{m} / \mathrm{z}=1432$, the expected value of the complexes formed between this charged lysozyme and the organic anion TPFB ${ }^{-}$were found. Complexes from 1:1 to 1:5 are observed for all major lysozyme charge states in the spectra.

interactions with Lys1, Arg14, and Arg128 as advocated for $\mathrm{TPBCl}^{-}$. This suggests a different mode of binding for $\mathrm{TPFB}^{-}$ or a mix of binding modes including the electrostatic interaction with the three cationic amino acids, supplemented by other binding locations.

BESI-MS with a Polarized Interface. The results from the capacitance potential scans suggest that potential differences lower than $\Delta_{0}^{\mathrm{w}} \phi=-0.05 \mathrm{~V}$ results in only a limited adsorption, whereas more positive potential differences result in a comparatively higher extent of adsorption. To test if this would also influence complexation, two additional BESI-MS experiments were conducted wherein the interface was chemically polarized to give either a more negative or more positive $\Delta_{0}^{\mathrm{w}} \phi$ compared to the original system. To do this a salt containing a water-soluble ion and a lipophilic ion were added to the aqueous phase. The lipophilic ion was identical to one of the ions in the oil phase, a so-called common ion. The common ion was either $\mathrm{TPBCl}^{-}$ or $\mathrm{BTPPA}^{+}$for both of which control experiments showed no detectable complexation with lysozyme when dissolved in the aqueous phase. In this way a distribution potential is established according to the Nernst equation. In the specific case of the common ion $\mathrm{TPBCl}^{-}$, the Nernst equation is written as

$$
\Delta_{\circ}^{\mathrm{w}} \phi=\Delta_{\circ}^{\mathrm{w}} \phi_{\mathrm{TPBCl}-}^{0}+\frac{R T}{z F} \ln \frac{a_{\mathrm{TPBCl}-}^{\mathrm{o}}}{a_{\mathrm{TPBCl}-}^{\mathrm{w}}}
$$

Where $\Delta_{0}^{\mathrm{w}} \phi$ is the distribution potential difference across the interface, $\Delta_{o}^{\mathrm{w}} \phi_{\mathrm{TPBCl}}^{0}$ is the standard ion transfer potential of $\mathrm{TPBCl}^{-}, z$ is the charge number of the ion and $a_{i}^{\alpha}$ is the activity of the ion $i$ in the phase $\alpha$. The concentration $(10 \mu \mathrm{M})$ of the common ion is chosen so as to be equal in both phases and thus the rightmost term cancels out under the assumption that concentration equals activity. The potential difference thus established equals the standard ion transfer potential of the common ion, in this case $\Delta_{o}^{\mathrm{w}} \phi_{\mathrm{TPBCl}}^{0}$. Because of the extreme lipophilicity of $\mathrm{TPBCl}^{-}$, the exact value of the standard ion transfer potential is unknown. However, it is known that it is higher than that for $\mathrm{H}^{+}, \Delta_{\circ}^{\mathrm{w}} \phi_{\mathrm{H}+}^{0}=0.53 \mathrm{~V},{ }^{44}$ and consequently $\Delta_{0}^{\mathrm{w}} \phi_{\text {TPBCl- }}^{0}>0.53 \mathrm{~V}$ leading to a potential difference of $\Delta_{o}^{w} \phi>$ $0.53 \mathrm{~V}$ according to eq 2 and under the assumption that $\Delta_{0}^{\mathrm{w}} \phi$ is not influenced by other ions. The BESI-MS spectrum for an interface that is positively polarized in this way is shown in Figure 6 (right). In the same way a positive potential can be chemically induced using a common lipophilic anion, so can a negative potential using a common lipophilic cation. Thus, a concentration of $10 \mu \mathrm{M} \mathrm{BTPPACl}$ in the aqueous and oil phase gives a potential difference of $\Delta_{0}^{\mathrm{w}} \phi=-0.70 \mathrm{~V}$, under the same assumptions as before, and by using the literature value of $\Delta_{o}^{w} \phi_{\mathrm{BTPA}+}^{0}{ }^{45}$ The results for the negatively polarized interface are shown in Figure 6 (left). With focus on the complexes formed with the $10+$ lysozyme the data from these experiments are compared (Table 1) to that of the interface that is not polarized.

It is evident that lowering the potential with respect to the open circuit potential difference, results in decreased complex formation, and vice versa for raising the potential. As discussed earlier, the complexes detected utilizing the BESI-MS method reflect events at the interface of the biphasic system. The results are in accordance with the capacitance potential scan (Figure 3), where the adsorption of protein to the interface was correlated with an interfacial potential difference. In this interpretation the lower potential difference results in less adsorption of lysozyme, as concluded from the small change in capacitance, and therefore less protein available for interacting at the interface. On the other hand, at highly positive potentials, more lysozyme is expected to accumulate at the interface which is in agreement with the increased amount of complexes observed.

(44) Sabela, A.; Marecek, V.; Samec, Z.; Fuoco, R. Electrochim. Acta 1992, 37, 231-35.

(45) Olaya, A. J.; Mendez, M. A.; Cortes-Salazar, F.; Girault, H. H. J. Electroanal. Chem. 2010, 644, 60-66. 

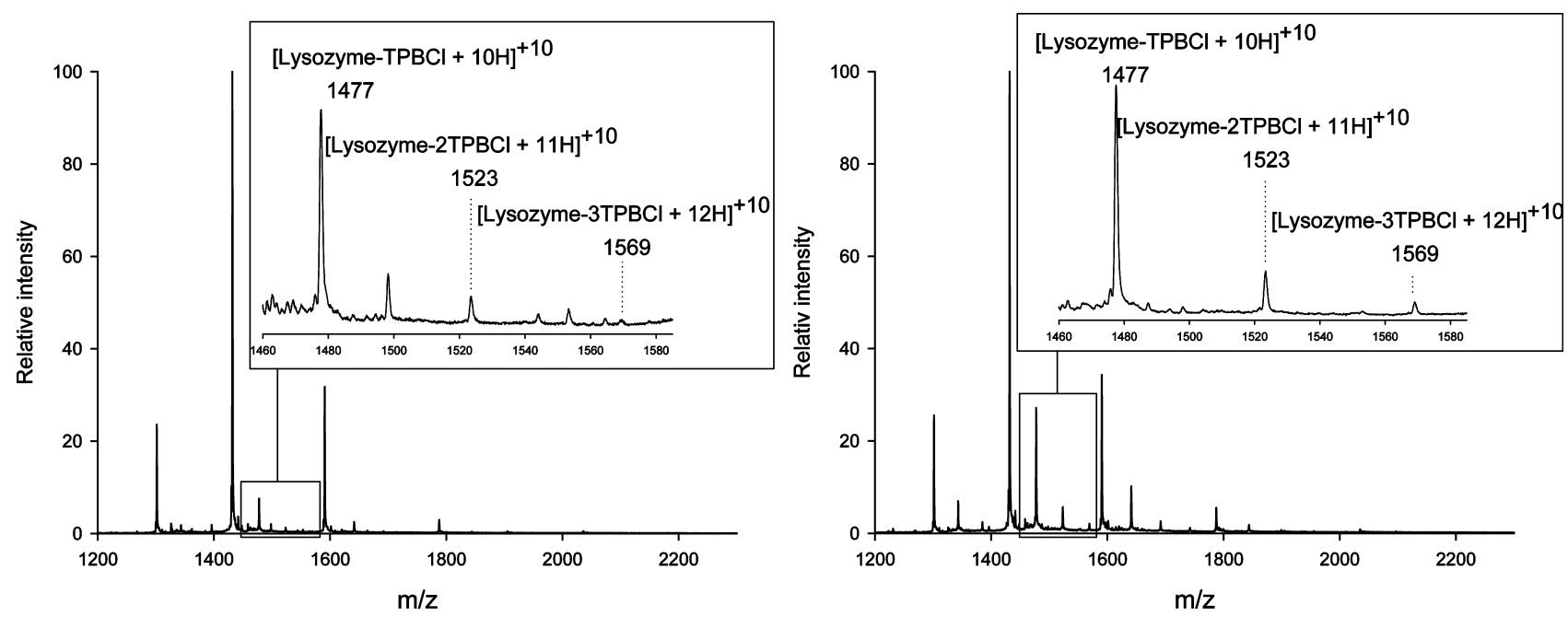

Figure 6. BESI-MS at chemically polarized interfaces. (left) an imposed negative potential difference between water and 1,2-DCE. Aqueous phase: $100 \mu \mathrm{M}$ Lysozyme and $10 \mu \mathrm{M} \mathrm{BTPPACl}$ in $0.5 \%(\mathrm{v} / \mathrm{v})$ acetic acid. Oil phase: $10 \mu \mathrm{M}$ BTPPATPBCI in DCE. 1:1, 1:2 and 1:3 complexes are observed at lower relative amounts than at open circuit potenital. (Right) an imposed positive potential difference between water and 1,2DCE. Aqueous phase: $100 \mu \mathrm{M}$ Lysozyme and $10 \mu \mathrm{M} \mathrm{KTPBCl}$ in $0.5 \%(\mathrm{v} / \mathrm{v})$ acetic acid. Oil phase: $10 \mu \mathrm{M}$ BTPPATPBCl in DCE. 1:1, 1:2, and $1: 3$ complexes are observed at higher relative amounts than at open circuit potential.

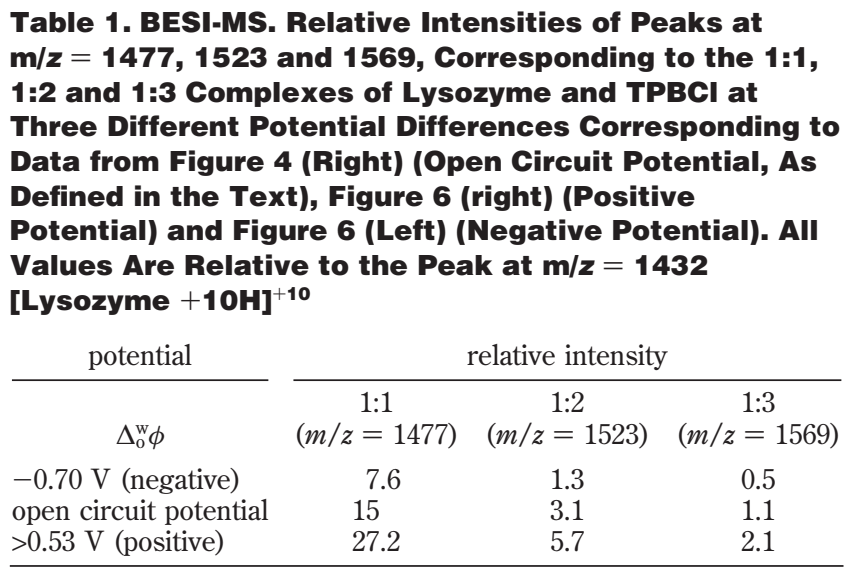

\section{CONCLUSIONS}

The present work investigated the interfacial interaction of the lipophilic ions $\left(\mathrm{TPBCl}^{-}\right.$and $\mathrm{TPFB}^{-}$) with the surface active protein lysozyme occurring at an oil-water interface. In addition, the effect of the interfacial potential difference on adsorption of lysozyme, and complex formation, at the oil-water interface was studied using a combination of electrochemical methods and BESI-MS. It was found that electrostatic interactions play a major part in the adsorption of lysozyme at the water-1,2-DCE interface, which is in accordance with its adsorption on many other surfaces. As a consequence it was possible to obtain partial control of the adsorption of the protein by controlling the interfacial potential. It was for the first time possible to directly detect a protein-ligand complex formed at an oil-water interface employing MS-detection and to study the dependence on an imposed interfacial potential difference on the complexation. It was shown that the formation of complexes specifically required the presence of the oil-water interface. From an analytical point of view the BESI-MS technique appears promising in different aspects of studying interactions at liquid-liquid interfaces: (i) as an analytical tool with all the possibilities that classical MS offer, for example, MS/MS could be used to discover the exact site on the protein where the anion interacts, (ii) as a potential tool to study interactions involving membrane proteins, which is, for example, important in the search for and characterization of drug targets, and (iii) for investigating the effect of an interfacial potential difference on complexation, which is likewise of interest when considering membrane proteins, as well as in relation to two phase drug delivery systems.

\section{ACKNOWLEDGMENT}

We acknowledge Carlsbergfondet for funding the Potentiostat/ Galvanostat PGSTAT30. R.A.H., L.J., M.W., J.Ø., and H.J. acknowledges the faculty of pharmaceutical sciences, University of Copenhagen for funding a "spirrekasse" project. The Fonds National Suisse pour la Recherche Scientifique is thanked for financial support through the project "Electrochemical methodology for the study of peptide lipid interaction” (Grant 200020113428).

Received for review June 9, 2010. Accepted August 13, 2010.

\section{AC101528R}

